\title{
Atypical psychopathology and clinical challenges in treating a patient with HIV-associated neurocognitive disorder
}

\author{
Balaswamy Reddy G. ${ }^{1}$, Guruprasad Srinivas ${ }^{2}$ \\ ${ }^{1}$ Department of Psychiatry, National Institute of Mental Health and Neurosciences (NIMHANS), Bengaluru, Karnataka, India \\ ${ }^{2}$ G.K. Reddy Neuropsychiatric Hospital, Kadapa, Andhra Pradesh, India
}

\begin{abstract}
Human immunodeficiency virus (HIV) has been known to be associated with primary central nervous system (CNS) manifestations in form of cognitive, behavioral, and affective symptoms. In the absence of opportunistic infections, the initial diagnosis of HAND (HIV-associated neurocognitive disorder) is difficult to suspect based on above in middle-aged adults, which commonly is misinterpreted as part of psychiatric disorders. Once HIV is diagnosed on serological testing, the personality changes are often colored by cognitive deficits induced by the illness itself, or because of antiretroviral treatment (ART) induced adverse effects, which can interfere with treatment compliance and worsening of HIV status. Acute onset alteration in mental status is known to happen with ART and HIV. With HIV, it can occur either because of primary CNS infection or opportunistic infections, whereas particular medications of ART were known to cause neuro-psychiatric manifestations ranging from anxiety, sleep disturbances, and short-lived increased dreams to psychosis. However, switching into aberrant states of consciousness with intense perceptual alterations for prolonged periods has rarely been reported. This case report highlights the importance of unique psychopathology and clinical helplessness in case of HAND, where cost-benefit of treatment change and psychoeducation of the family was a challenge.
\end{abstract}

HIV AIDS Rev 2018; 17, 3: 224-226 DOI: https://doi.org/10.5114/hivar.2018.78496

Key words: HIV, neurocognitive, atypical.

\section{Introduction}

Human immunodeficiency virus (HIV) is known to be associated with a variety of psychiatric manifestations ranging from substance use (40-74\%), depression (22-50\%), anxiety (2-40\%), bipolar disorder, suicide, obsessive-compulsive disorder (OCD) to psychosis [1, 2]. For each of above, the list of differential diagnoses will be compounded by the bidirectional link between HIV and mental illness, chronicity of the illness, significant losses and isolation, and complex medical treatments in HIV, comparing to general population $[3,4]$.

Address for correspondence: Dr. Balaswamy Reddy G., MD, Department of Psychiatry, National Institute of Mental Health and Neurosciences (NIMHANS), Bengaluru, Karnataka-560029, India, e-mail: gbalambbs7819@gmail.com

However, the occurrence of psychosis is common among HIV-infected people than the general population due to a variety of reasons like chance associations, genetic predisposition, HIV brain infection, opportunistic infection (OI), or because of antiretroviral therapy (ART) itself, it is uncommon to compare it with affective disorders with prevalence around $1 \%$. Psychosis, most of the times, occurs due to organic causes like delirium, OI like toxoplasma, Cryptococcus, or rarely progressive multifocal leukoencephalopathy (PML) [5-7]. Acute onset alteration in mental status is known to happen both with HIV and ART but prolonged

Article history:

Received: 22.10 .2017

Received in revised form: 08.01.2018

Accepted: 10.01.2018

Available online: 15.08 .2018
International Journal of HIV-Related Problem

HIV \& AIDS

$R$ e vi e w 
perceptual abnormalities and daydreaming have rarely been reported in central nervous system (CNS)-HIV.

Here, we are presenting a case, which highlights the importance of unique psychopathology, diagnostic difficulties, and challenges in treatment.

\section{Case report}

A 39-years-old male was presented with a diagnosis of HIV and bipolar disorder. The patient was on ART (lamivudine, efavirenz, and tenofovir) over the past 1 year, along with valproate $1,000 \mathrm{mg}$ and risperidone $4 \mathrm{mg}$.

He had a 4 years history of insidious onset, progressive change in his personality with mood dysregulation, apathy, pseudo-philosophical ideas, ideational perseveration, and hoarseness of voice. One year back while evaluating speech changes, pre-surgical ELISA tests for laryngeal biopsy was ordered and the results revealed HIV-positive status of the patient, with a CD4 count of 143 . The suspected mode of transmission was through tattoos, as his sexual partners were seronegative status.

The patient was referred to the ART center and started ART therapy, along with co-trimoxazole prophylaxis. A month after starting ART, the CD4 count showed improvement (190); however, his attention and concentration were significantly worsened, and started experiencing altered states of consciousness. He would switch into daydreaming spontaneously and become completely aware of the fact. These symptoms were almost similar to the picture of an oneiric state. Reduction in environmental stimuli would cause to transform the patient into a vivid dream-like state, where he would see and feel the abnormal perceptual experience and started attributing it to meditation. He was aware that all his experiences were not true and at the same time, he felt extremely distressed and helpless due to inability to control them. Also, he became aware of blood flow in hands and perceived it like electric current sensations, experienced as extreme power and ability to cure others, which qualified for coenaesthesia.

The patient's compliance to medications was dropped due to inability to control over his perceptual experiences and apparent feeling of treatment being not helpful. Physical examination: bald tongue, mild hepatomegaly, husky voice, cerebellar signs positive, exaggerated deep tendon reflexes.

Mental status examination: psychomotor slowing, thought retardation, abnormal perceptual experiences, which were as described above, including spontaneous slippage into vivid dreamy state of consciousness, associated with reduction in the external stimuli in the absence of disorientation and ability to distinguish it from reality, memory deficits in immediate (registration, delayed recall), and recent memory, impaired arithmetic and abstract thinking. Additional clinical/laboratory evaluation included as shown in the Table 1.

Patient's final diagnosis was made as HAND (HIV-associated neurocognitive disorder) and psychosis due to primary HIV or ART-induced. His risperidone $4 \mathrm{mg}$ was cross tapered with quetiapine $400 \mathrm{mg}$, in view of extrapyramidal
Table 1. Summary of clinical/laboratory work-up

\begin{tabular}{l|c}
\hline Assessments & Results \\
\hline Blood investigations & $\begin{array}{c}\text { Decreased vitamin } B_{12} \text { levels, } \\
\text { dyslipidemia, HIV-1 type virus, } \\
\text { CD4 count 273/mc }\end{array}$ \\
\hline IHDS & $8 / 12$ \\
\hline MoCA & $16 / 31$ \\
\hline HMSE & $23 / 31$ \\
\hline MMSE & $26 / 30$ \\
\hline Clinical criteria for HAND* & Satisfied \\
\hline Neuropsychology & $\begin{array}{c}\text { B/L DLPFC, ACC, right medial } \\
\text { temporal and fronto-parietal } \\
\text { areas involved }\end{array}$ \\
\hline MRI brain & Normal \\
\hline
\end{tabular}

IHDS - International HIV Dementia Scale, MoCA - Montreal Cognitive

Assessment, HMSE - Hindi Mental Status Examination, MMSE - Mini Mental Status Examination, B/L DLPFC - bilateral dorsal lateral prefrontal cortex, $A C C$ - anterior cingulate cortex *[2]

symptoms and noted to have improvement in slowness, irritability, and coenesthetic experiences, but there was no change in his dream-like states, where he had no control over it. For an expert opinion on the ART regimen, he was referred to apex ART center, New Delhi. On a telephonic follow-up after 4 months, reportedly efavirenz was changed to nevirapine in his ART regimen, along with quetiapine dose to $800 \mathrm{mg}$ /day with no further improvement.

\section{Discussion}

Occasionally, HIV-associated psychiatric illness can present a diagnostic challenge, when HIV was not diagnosed initially, as in this case, and treated as a bipolar illness for the initial symptoms. The above case description is unique for several reasons including uncommon mode of transmission, atypical perceptual abnormalities, primary CNS HIV manifestation without any evidence of OI, prolonged altered state of consciousness in the absence of delirium or OI, and finally, no significant imaging findings and clinical helplessness in treating the patient.

Oneroid states are a kind of aberrant states of consciousness, where the person experiences fantastic hallucinations, illusions with affective responses, become perplexed, often disoriented, feels and behaves as though in a dream. These are mostly short limited, known to occur in schizophrenic breakdowns as per the literature. However, in case of HIV, no author has reported on such states. Additionally, in this case, the patient is oriented and arousable during the episodes of the dreamy state, fully aware of those perceptual alterations.

In his case, whether HIV infection, ART, or both contributed to the above symptomatology is not clear. Structurally, gray matter atrophy of cerebral cortex, particularly in ACC, lateral temporal cortex, primary motor and sensory cortices, frontal and parietal lobes, and white matter abnormalities 
are expected to be as per the literature [8-12]: In the above case, there were no significant findings on imaging and no evidence of OI, as OI like toxoplasmosis or Cryptococcosis are known to cause psychosis.

Although, efavirenz is the initial regimen recommended by WHO guidelines and has good CSF penetration factor, there is consistent evidence showing that efavirenz can cause psychiatric symptoms like nightmares or vivid dreams, however mostly short-lived (2-6 weeks) as per a meta-analysis by Kenedi et al. [12-14]. No findings on prolonged and persistent oneroid states were described [14-16]. There are few case reports relating efavirenz to psychosis [17-20], suggesting that efavirenz in combination with tenofovir increases the risk of psychosis [21].

Challenges of the treatment were concerns about drug interactions, limited literature on the use of antipsychotics, and increased risk for EPS [22]. Since risperidone has not benefited for the patient, quetiapine was prescribed with partial improvement in psychotic symptoms, but no response was noted in patient's altered dreamlike-states. The clinical presentation of abnormal perceptual psychopathology in this patient could be attributed to either of these or both - primary CNS-HIV infection or efavirenz treatment. This case report demonstrates clinical challenges related to atypical presentation and multiple factors contributing to the clinical presentation in organic disorders.

\section{Conflict of interest}

The authors declare no potential conflicts of interest with respect to the research, authorship, and/or publication of this article.

\section{References}

1. Allavena C, Le Moal G, Michau C, et al. Neuropsychiatric adverse events after switching from an antiretroviral regimen containing efavirenz without tenofovir to an efavirenz regimen containing tenofovir: a report of nine cases. Antivir Ther 2006; 11: 263-265.

2. Bonnet F, Amieva H, Marchant F, et al.; S CO3 Aquitaine Cohort. Cognitive disorders in HIV-infected patients: are they HIV-related? AIDS 2013; 27: 391-400.

3. Dubé B, Benton T, Cruess DG, Evans DL. Neuropsychiatric manifestations of HIV infection and AIDS. J Psychiatry Neurosci 2005; 30: 237-246.

4. Foster R, Olajide D, Everall IP. Antiretroviral therapy-induced psychosis: case report and a brief review of the literature. HIV Med 2003; 4: 139-144.

5. Hinsch MC, Reichelt D, Husstedt IW. Acute psychosis as a side effect of efavirenz therapy with metabolic anomalies: an important differential diagnosis of HIV-associated psychoses. Nervenarzt 2014; 85: 1304-1308.

6. Jayarajan N, Chandra PS. HIV and mental health: An overview of research from India. Indian J Psychiatry 2010; 52 (Suppl 1): S269-S273.

7. Kenedi CA, Goforth HW. A systematic review of the psychiatric side-effects of efavirenz. AIDS Behav 2011; 15: 1803-1818.

8. Kumar P, Jain MK. Progressive multifocal leukoencephalopathy in AIDS camouflaged with catatonia: A wolf in sheep's clothing. Indian J Psychiatry 2006; 48: 69-71.

9. Küper M, Rabe K, Esser S, et al. Structural gray and white matter changes in patients with HIV. J Neurol 2011; 258: 1066-1075.
10. De la Garza LC, Paoletti-Duarte S, García-Martín C, GutiérrezCasares JR. Efavirenz-induced psychosis. AIDS 2001; 15: 1911-1912.

11. Lowenhaupt EA, Matson K, Qureishi B, et al. Psychosis in a 12-yearold HIV-positive girl with an increased serum concentration of efavirenz. Clin Infect Dis 2007; 45: e128-130.

12. Manning TG. Efavirenz and psychosis: is there a link? Aust NZJ Psychiatry 2012; 46: 687-688.

13. Nebhinani N, Mattoo SK. Psychotic Disorders with HIV Infection: A Review. German J Psychiatry 2013; 16: 43-48.

14. Nedelcovych MT, Manning AA, Semenova S, et al. The Psychiatric Impact of HIV. ACS Chem Neurosci 2017; 8: 1432-1434.

15. Neuropsychiatric aspects of HIV infection among older adults. Bull World Health Organ 1990; 68: 671-681.

16. Oudijn MS, Vrijlandt CM, Casteelen G. Severe psychosis in an African woman due to the antiretroviral agent efavirenz. Ned Tijdschr Geneeskd 2006; 150: 643-644.

17. Poulsen HD, Lublin HK. Efavirenz-induced psychosis leading to involuntary detention. AIDS 2003; 17: 451-453.

18. Ragin AB, Du H, Ochs R, et al. Structural brain alterations can be detected early in HIV infection. Neurology 2012; 79: 2328-2334.

19. Steinbrink F, Evers S, Buerke B, et al. German Competence Network HIV/AIDS. Cognitive impairment in HIV infection is associated with MRI and CSF pattern of neurodegeneration. Eur J Neurol 2013; 20: 420-428.

20. Sütterlin S, Vögele C, Gauggel S. Neuropsychiatric complications of efavirenz therapy: suggestions for a new research paradigm. J Neuropsychiatry Clin Neurosci 2010; 22: 361-369.

21. David A, Fleminger S, Kopelman M, et al. Lishman's Organic Psychiatry: A Textbook of Neuropsychiatry. $4^{\text {th }}$ ed. http://www.wiley.com/ WileyCDA/WileyTitle/productCd-0470675071.html (Accessed: 25 August 2017). 